\title{
Review of: "Excitatory synapses and gap junctions cooperate to improve Pv neuronal burst firing and cortical social cognition in Shank2-mutant mice"
}

Jose Bargas $^{1}$

1 Universidad Nacional Autónoma de México

Potential competing interests: The author(s) declared that no potential competing interests exist.

\section{Of mice and neurons making networks}

José Bargas

Departamento de Neurociencias. Instituto de Fisiología Celular, Universidad Nacional Autónoma de México. Mexico City. Mexico.

The prefrontal cortex (PFC) is key to control executive behaviors such as: cognition, attention, working memory, social interaction and emotion (Bartos et al., 2007). The ordered and coordinated function of cortical circuits, seen as the sequential activation of neuronal ensembles aligned with behavior, depends on the action of fast spiking interneurons, which express parvalbumin (FS-PV interneurons; Sohal et al., 2009; Serrano-Reyes et al., 2020). FS-PV interneurons preferentially target perisomatic postsynaptic sites and axonal initial segments. They are known by their firing at high frequencies, bursting, brief action potentials, as well as their reciprocal and specific connections with different networks of pyramidal neurons (PN; Kawaguchi et al., 2019), generating a potent inhibition to stop firing or, on the contrary, to synchronize firing during hyperpolarizing rebounds, thus having a key role in the excitatory/inhibitory balance required for the proper function of any brain circuit (Ferguson \& Gao, 2018). Their contribution to the generation of gamma frequency rhythms in the brain (Bartos et al., 2007) is correlated with attention, cognition and information processing and transfer. In consequence, disorders in their function lead to PFC dysfunction and psychiatric disease.

Among the important psychiatric ailments related to PFC and FS-PV interneurons are the autism spectrum disorders (ASD). However, the specific failures in the properties of these neurons that make them potential candidates as therapeutic targets to treat ASD are in debate. In the age of social networking, when interactions between individuals are essential to accomplish social collaboration and goals, ASD call our attention with particular relevance.

In the present commented work, the authors (Lee et al., 2021) use behavioral, in vitro and in vivo electrophysiological recordings, optogenetical and computational experiments in a mice model of ASD: the PV-Cre;Shank ${ }^{2-/-}$ or Shank2-/- mice, generated by crossing Shank ${ }^{2-/-}$ mice with PV-Cre mice and transfected them with AAV-Dio-ChR2-EYFP in the MPFC to generate FS-PV neuron-specific ChR2 expression 
for photostimulation.

Electrophysiological recordings of both units and local field potentials (LFP) were done in the medial PFC (mPFC). Compared with wild type (WT) subjects, Shank KO mice showed a decrease in FS-PV interneurons activity linked to object and social target discrimination, a significant decrease in burst firing and a significant decrease in the power of oscillations during social target encounters, in particular in the gamma range, even when oscillatory power is higher during resting periods. By light stimulating these neurons the authors found that FS-PV interneurons failed to inhibit PN in Shank KO mice, while inhibition in WT mice was present as normal. Inhibition of PN is essential to generate gamma oscillations (Bartos et al., 2007). Thus, optogenetic experiments clearly demonstrate a failure in this mechanism in ASD subjects. Importantly, this happens without changes in the biophysical properties, input-output curves, excitability or baseline synaptic transmission of both FS-PV interneurons and PN.

How to explain the decrease in burst firing and oscillatory power? Are these findings associated to a lack of proper social behavior? Previous findings (Won et al., 2012; Saunders et al., 2013) showed that Shank KO were deficient in NMDAR function as measured with the NMDA/AMPA ratio as compared with WT mice. This deficiency explains in part low burst firing and ADS in Shank KO mice. Indeed, the treatment with a NMDAR receptor enhancer, D-cycloserine (a glycine agonist on NMDAR), or a metabotropic glutamate receptor agonist, increased bursting in both Shank KO and WT animals improving social behavior in Shank KO mice. Interestingly, this increase was blocked by mefloquine, an antagonist of gap junctions (G); Lee et al., 2021), suggesting that NMDAR deficiency also decreases GJ activity. FS-PV interneurons are known to form a network by way of GJ and chemical inhibitory synapses (Connors et al., 2017) increasing circuit capabilities, and the present work shows that NMDAR dysfunction is also present in FS-PV interneurons. Given these antecedents, the authors tested light stimulation on FS-PV interneurons from Shank KO mice. This procedure increased bursting and gamma rhythms when the stimulus was delivered at $10 \mathrm{~Hz}$, not at 40 $\mathrm{Hz}$ as expected (even when using CheTA or SSFO), further suggesting the involvement of GJ due to their low band pass filtering. Focused somatic photostimulation of ChR2 expressing FS-PV interneurons also exhibited a robust burst firing in neighboring PV neurons as compared with WT interneurons even in the presence of synaptic blockers, confirming the involvement of GJ networking in this process. In fact, GJ activity in Shank KO interneurons was higher than in WT interneurons. Computational modeling experiments then showed that communication between FS-PV interneurons was increased between Shank KO bursting neurons. Number and strength of GJ in FS-PV interneurons was positively correlated with the increase in burst firing in stimulated and neighboring FS-PV interneurons. Together with bursting increases, photostimulation of FS-PV interneurons improved cortical social representation and social behavior in Shank KO mice. Bursting and GJ networking of FS-PV interneurons had been somehow neglected in previous studies of autism.

Hence, the work by Lee et al (2021), metaphorically shows that the "social" networking of FS-PV interneurons is essential for the proper function of PFC, and therefore, social interactions and behavior of autistic individuals. To make the image explicit: a regulated FS-PV interneurons networking is essential for 
the social networking of their mice owners.

Nevertheless, it would be hard that a complex interaction between NMDAR and GJ has no confounds. Interestingly, direct simulation of Shank KO FS-PV interneurons did not increase their burst firing upon Dcycloserine treatment, suggesting that GJ need to cooperate with NMDAR to promote FS-PV interneuron bursts to be effective in networking. Moreover, knocking out GJ in WT mice shows that, by themselves, the hyperactivity of GJ has negative influences in social interaction, and it is in the context of the already disrupted social interaction of Shank2/- mice, when GJ hyperactivity is associated with NMDAR hypoactivity to suppress burst firing. It is in this case when FS-PV photostimulation has its positive actions on social behavior. Perhaps not by just potentiating the FS-PV interneurons electrical network but by regulating its proper function in a way that needs further investigation.

\section{REFERENCES}

Bartos, M., Vida, I. \& Jonas, P. (2007). Synaptic mechanisms of synchronized gamma oscillations in inhibitory interneuron networks. Nat Rev Neurosci 8: 45-56. doi.org/10.1038/nrn2044.

Connors BW. (2017). Synchrony and so much more: Diverse roles for electrical synapses in neural circuits. Dev Neurobiol 77: 610-624. doi: 10.1002/dneu.22493.

Ferguson BR, Gao WJ. (2018). PV Interneurons: Critical Regulators of E/I Balance for Prefrontal CortexDependent Behavior and Psychiatric Disorders. Front Neural Circuits. 12: 37. doi:

\subsection{9/fncir.2018.00037}

Kawaguchi Y, Otsuka T, Morishima M, Ushimaru M, Kubota Y. (2019). Control of excitatory hierarchical circuits by parvalbumin-FS basket cells in layer 5 of the frontal cortex: insights for cortical oscillations. J Neurophysiol 121: 2222-2236. doi: 10.1152/jn.00778.2018.

Lee E, Lee S, Shin JJ, Choi W, Chung C, Lee S, Kim J, Ha S, Kim R, Yoo T, Yoo YE, Kim J, Noh YW, Rhim I, Lee SY, Kim W, Lee T, Shin H, Cho IJ, Deisseroth K, Kim SJ, Park JM, Jung MW, Paik SB, Kim E. (2021). Excitatory synapses and gap junctions cooperate to improve Pv neuronal burst firing and cortical social cognition in Shank2-mutant mice. Nat Commun 12: 5116. doi: 10.1038/s41467-021-25356-2.

Saunders JA, Tatard-Leitman VM, Suh J, Billingslea EN, Roberts TP, Siegel SJ. (2013). Knockout of NMDA receptors in parvalbumin interneurons recreates autism-like phenotypes. Autism Res 6: 69-77. doi: 10.1002/aur.1264.

Serrano-Reyes M, García-Vilchis B, Reyes-Chapero R, Cáceres-Chávez VA, Tapia D, Galarraga E, Bargas J. (2020). Spontaneous Activity of Neuronal Ensembles in Mouse Motor Cortex: Changes after GABAergic Blockade. Neuroscience 446: 304-322. doi: 10.1016/j.neuroscience.2020.08.025.

Sohal VS, Zhang F, Yizhar O, Deisseroth K. (2009). Parvalbumin neurons and gamma rhythms enhance cortical circuit performance. Nature 459: 698-702. doi: 10.1038/nature07991.

Won H, Lee HR, Gee HY, Mah W, Kim Jl, Lee J, Ha S, Chung C, Jung ES, Cho YS, Park SG, Lee JS, Lee K, Kim D, Bae YC, Kaang BK, Lee MG, Kim E. (2012). Autistic-like social behaviour in Shank2-mutant mice improved by restoring NMDA receptor function. Nature 486: 261-265. doi: 10.1038/nature11208. 
FINANCING: FORDECYT-PRONACES 154039 